\title{
TFT Small Signal Model and Analysis
}

\author{
Xiang Cheng, Student Member, IEEE, Sungsik Lee, Member, IEEE, and Arokia Nathan, Fellow, IEEE
}

\begin{abstract}
We present an accurate small signal model for thin film transistors (TFTs) taking into account non-idealities such as contact resistance, parasitic capacitance, and threshold voltage shift. The model gives high accuracy in s-parameters, and the predicted cutoff frequency yields $1 \%$ discrepancy compared with measurement results. In contrast, the conventional CMOS small signal model adapted for TFTs yields $12.5 \%$ error. The TFT's cutoff frequency is also evaluated under bias stress to examine the effect of device instability on small signal behavior.
\end{abstract}

Index Terms-Small signal model, s-parameter, thin film transistor (TFT), cutoff frequency, threshold voltage $\left(V_{T}\right)$ shift

\section{INTRODUCTION}

$S_{\mathrm{d} e}^{\mathrm{m}}$ mall signal modeling simplifies the analysis of non-linear devices, in which the signal in question is treated as a small perturbation around the bias point [1]-[3]. The resulting linear approximation reduces design complexity of circuits such as amplifiers [4]-[9]. When compared to MOSFETs, TFTs have a different structure and unique material properties, which result in, for example, larger contact resistance, high parasitic capacitance, and stress-induced $\mathrm{V}_{\mathrm{T}}$ shift [10]-[20]. This is true for a-Si:H [10]-[12], organic [13]-[17] and metal-oxide [18][20] TFTs alike. Therefore, it is imperative that these non-idealities be captured in the small signal model. This is especially crucial in TFT-based analog circuit design and in particular, for the accurate prediction of the circuit's dynamic behavior.

In this letter, we present an accurate TFT small signal model where the contact resistance $\left(\mathrm{R}_{\mathrm{C}}\right)$, parasitic capacitance, and stress induced threshold voltage $\left(\mathrm{V}_{\mathrm{T}}\right)$ shift are considered. The small signal behavior is analyzed through measurements of S-parameters, current-voltage (I-V) and capacitance-voltage $(\mathrm{C}-\mathrm{V})$ characteristics, and bias-induced instability. From these measurements, we assess if the MOSFET's small signal model is adequate or examine the modifications needed for TFTs using a-InGaZnO (IGZO) test TFT structures.

\section{THEORETICAL ANALYSIS}

The TFT structure examined here and equivalent passive components are shown in Fig. 1(a), in which the transistor is working in the saturation regime, where the bias conditions follow $V_{D S}>V_{G S}>V_{T}$. The shaded region indicates the formed

Manuscript received, xxx.2015. This work is partly supported by the project XXXX. X. Cheng, S. Lee, and A. Nathan are with Electrical Engineering Division, Department of Engineering, University of Cambridge, Cambridge CB3 0FA, U.K. (e-mail: xc260@cam.ac.uk; s1684@cam.ac.uk; an299@eng.cam.ac.uk).

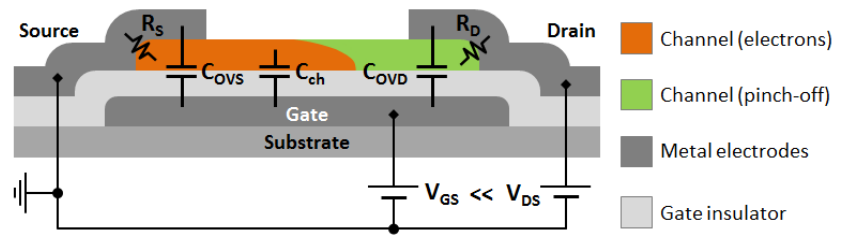

(a)

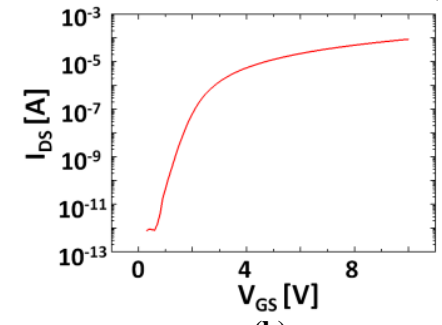

(b)

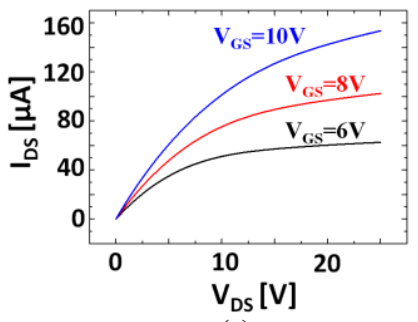

(c)
Fig. 1. (a) Bottom gate TFT structure and equivalent passive components, where $\mathrm{C}_{\mathrm{ch}}$ is the channel capacitance, $\mathrm{C}_{\mathrm{OVS} / \mathrm{ovD}}$ the overlap capacitance at source/drain side, $\mathrm{R}_{\mathrm{S} / \mathrm{D}}$ the contact resistance at source/drain side. (b) Transfer characteristics (c) output characteristic of the TFT under test. Test structures used for model synthesis were IGZO TFTs reported in [23], [26]. The device under test has following physical and geometrical parameters: $t_{\mathrm{s}}=50 \mathrm{~nm}$, $\mathrm{V}_{\mathrm{T}}=1.6 \mathrm{~V}, \mathrm{C}_{\mathrm{OX}}=30 \mathrm{nF} / \mathrm{cm}^{2}, \mu=8.6 \mathrm{~cm}^{2} / \mathrm{Vs}$

channel and pinch-off happens at the edge adjacent to the drain electrode. As illustrated, the channel capacitance only contributes to the source side since the drain side is pinched off. The corresponding small signal equivalent circuit model is shown in Fig. 2(b), where the contact resistances and overlap capacitances are connected. The working channel in saturation is then represented by a voltage controlled current source in parallel with an output resistance $\left(r_{0}\right)$, which is supported by Thévenin's theorem [21], [22] since other capacitance or inductance effects are negligible.

This approach takes a different path from the CMOS small signal model (see also Fig. 2). In particular, the contact resistance separates the channel capacitor $\left(\mathrm{C}_{\mathrm{ch}}\right)$ and the overlap capacitor at the source side $\left(\mathrm{C}_{\mathrm{OVS}}\right)$, which would yield a different frequency response, especially for the $S_{11}$ parameter. In addition, the transconductance $\left(\mathrm{g}_{\mathrm{m}}\right)$ in the TFT model seen in Fig. 2(b) is no longer in linear operation as a function of the bias voltage. Note that the connection of contact resistance and $\mathrm{C}_{\mathrm{ch}}$ are also valid for other material families and device structures.

\section{A. Small signal model at low frequency}

At very low frequencies, all the parasitic capacitances and channel capacitances can electrically be treated as open-circuit connections. This is valid when the frequency of concern is much smaller than the first pole of the frequency response (i.e. $f \ll f_{p} \approx 1 / 2 \pi R_{L} C_{O V D}$, where $\mathrm{R}_{\mathrm{L}}$ is the load resistance connected at the drain of the TFT, assuming no parasitic at input). In this frequency regime, the TFT model can be 
equivalent to the CMOS model omitting all the capacitors according to Thévenin's theorem. Note that the $\mathrm{g}_{\mathrm{m}}$ and $\mathrm{r}_{\mathrm{o}}$ in Fig. 2(a) can be calculated directly from the derivative of the measured drain current vs. gate-source voltage (i.e. $\mathrm{I}_{\mathrm{D}}-\mathrm{V}_{\mathrm{GS}}$ ) and drain current vs. drain-source voltage (i.e. $\mathrm{I}_{\mathrm{D}}-\mathrm{V}_{\mathrm{DS}}$ ) curves, respectively, while $g_{\mathrm{mi}}$ and an internal output resistance $\left(\mathrm{r}_{\mathrm{o}}\right)$ cannot be obtained directly from the static behavior. As both models should capture the same derivatives of the static behavior, the expressions for $\mathrm{g}_{\mathrm{mi}}$ and $\mathrm{r}_{\mathrm{oi}}$ as a function of measurable parameters can be derived as follows:

$$
\begin{gathered}
g_{m i}=\frac{g_{m} r_{o}}{r_{o}-g_{m} r_{o} R_{C}-2 R_{C}} \\
r_{o i}=r_{o}-g_{m} r_{o} R_{C}-2 R_{C}
\end{gathered}
$$

Here, $\mathrm{R}_{\mathrm{C}}=\mathrm{R}_{\mathrm{D}}=\mathrm{R}_{\mathrm{S}}$, assuming the contact properties at source and drain sides are symmetrical.

According to the static models developed in [23] based on the same set of samples, the internal $g_{m i}$ (Fig. 2(b)) considering $V_{T}$ shift can be expressed as:

$$
\begin{aligned}
g_{m i} & =K \frac{W}{L}\left(V_{G A}-V_{T}-\Delta V_{T}\right)^{\alpha+1} \\
& \approx K \frac{W}{L}\left(V_{G A}-V_{T}\right)^{\alpha+1}-K \frac{W}{L}(\alpha+1)\left(V_{G A}-V_{T}\right)^{\alpha} \Delta V_{T} \\
& =g_{m i 0}-K^{\prime} \Delta V_{T}
\end{aligned}
$$

where $\mathrm{g}_{\operatorname{mi0}}$ is the initial value without any $\mathrm{V}_{\mathrm{T}}$ shift, $K^{\prime}=$ $K(W / L)(\alpha+1)\left(V_{G A}-V_{T}\right)^{\alpha}$, as $\alpha$ is a constant close to zero, $K^{\prime}$ is weakly dependent on bias and can be treated as a constant. The $\left|\Delta \mathrm{V}_{\mathrm{T}}\right|$ can be represented with the following stretched exponential functions for stress and recovery stages, respectively, as $\left|\Delta V_{T}\right|=\left(V_{G S}-V_{T}\right)\left(1-\exp \left(-\left(t / \tau_{S}\right)^{\beta_{S}}\right)\right)$ and $\left|\Delta V_{T}\right|=\Delta V_{T s t} \exp \left(-\left(t / \tau_{R}\right)^{\beta_{R}}\right)$ [10], [18]. Here, $\Delta \mathrm{V}_{\text {Tst }}$ is the initial value of $\Delta \mathrm{V}_{\mathrm{T}}$ at the start of recovery stage, $\tau_{\mathrm{S}}$ and $\tau_{\mathrm{R}}$ are time constants for stress and recovery stages, respectively, and $\beta_{\mathrm{S}}$ and $\beta_{\mathrm{R}}$ are exponents for stress and recovery stages, respectively. These parameters are different relating to stress conditions, such as bias stress and illumination stress [10], [18], [24]. The assumption of $\Delta \mathrm{V}_{\mathrm{T}}<<\mathrm{V}_{\mathrm{GS}} \mathrm{V}_{\mathrm{T}}$ is used in the approximation of Eq. (3).

The derivation above shows that the $\mathrm{V}_{\mathrm{T}}$ shift can be represented by a separate component. The corresponding small-signal model is shown in Fig. 2(b).

\section{B. Small signal model at high frequency}

At higher frequencies, capacitance effects are no longer negligible (i.e. when the low frequency assumption in the above section is violated), and the small signal model becomes different from the CMOS counterpart. This means that the two models in Fig. 2 should give different cut-off frequencies and different bode-plots for the current gain $\left(\mathrm{H}_{21}\right)$.

The short circuit current gain $\left(\mathrm{A}_{\mathrm{i}}\right)$ calculated from the CMOS model in Fig.2(a) is represented as,

$$
A_{i}=\frac{s C_{O V D}-g_{m}}{s\left(C_{O V D}+C_{o V S}+C_{c h}\right)}
$$

In the bode plot for Eq.(4), there should be one pole and zero. And the cutoff frequency $\left(\mathrm{f}_{\mathrm{T}}\right)$ is then approximated by assuming that the zero of $\mathrm{g}_{\mathrm{m}} / \mathrm{C}_{\mathrm{OVD}}$ is far from the point of the cutoff frequency. Thus,

$$
f_{T}=\frac{g_{m}}{2 \pi\left(C_{O V D}+C_{o v S}+C_{c h}\right)}=\frac{g_{m}}{2 \pi C_{t o t}}
$$

(a)

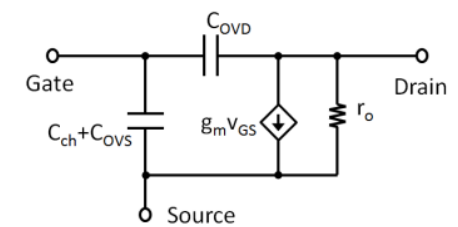

(b)

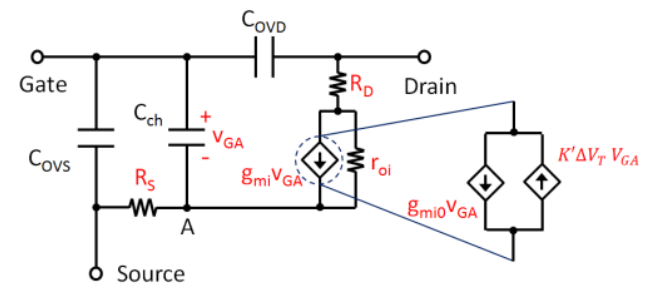

Fig. 2. (a) CMOS small signal model and (b) TFT model. Here, $V_{G A}$ is the voltage difference between gate and the node 'A', where $V_{G A}=V_{G S}-I_{D S} R_{S}$

where $\mathrm{C}_{\text {tot }}$ is the sum of all capacitances of the transistor.

In contrast, the current gain calculated for the TFT small signal model seen in Fig.2(b) is

$$
A_{i}=\frac{-g_{m i} r_{o 1}+\left(\left(2 R_{C}+r_{o i}+g_{m i} R_{C} r_{o i}\right) C_{O V D}+C_{C h} R_{C}\right) s+C_{O V D} C_{c h} R_{C}\left(R_{C}+r_{o i}\right) s^{2}}{s\left(g_{m i} C_{O V} R_{C} r_{o i}+\left(2 R_{C}+r_{o i}\right)\left(C_{O V}+C_{c h}\right)+C_{O V}\left(R_{C}+r_{o i}\right) C_{c h} s\right)}
$$

where $C_{O V}=C_{O V S}+C_{O V D}$. Therefore the cut-off frequency is:

$$
f_{T}=\frac{g_{m i} r_{o i}}{2 \pi\left(g_{m i} C_{O V} R_{C} r_{o i}+\left(2 R_{C}+r_{o i}\right)\left(C_{o V}+C_{c h}\right)\right)}=\frac{g_{m}}{2 \pi\left(C_{t o t}-g_{m} R_{C} C_{c h}\right)}
$$

From Eqs.(5) and (7), we can clearly see that the major difference between Figs.2(a) and (b) comes from the term $\mathrm{g}_{\mathrm{m}} \mathrm{R}_{\mathrm{C}} \mathrm{C}_{\mathrm{ch}}$. This implies that this difference depends on the value of $R_{C}$ and the fraction $C_{c h}$ is of the total capacitance. Note that $\mathrm{C}_{\mathrm{ch}}$ is related to both free and trapped charges associated with the degree of disorder in the channel layer [25], [26]. This also indicates that the CMOS model can be inaccurate especially when the contact resistance and overlap capacitance are big and small, respectively.

\section{RESUlTS AND DisCUSSIONS}

The s-parameters are measured using the Keysight E5061B network analyzer calibrated by CS-11 calibration substrate provided by GGB Industries, Inc. The $\mathrm{DC}$ bias is at $\mathrm{V}_{\mathrm{GS}}=8 \mathrm{~V}$ and and $\mathrm{V}_{\mathrm{DS}}=15 \mathrm{~V}$. The cut-off frequency extracted from the converted $\mathrm{H}_{21}$ parameter yields the result shown in Fig. 3. The extracted device parameters used here are as follows: $\mathrm{g}_{\mathrm{m}}=16.5$ $\mu \mathrm{S}, \quad \mathrm{C}_{\mathrm{ch}}=0.2 \mathrm{pF}, \quad \mathrm{C}_{\mathrm{OVD}}=\mathrm{C}_{\mathrm{OVS}}=0.43 \quad \mathrm{pF}, \quad \mathrm{r}_{\mathrm{o}}=1.4 \mathrm{M} \Omega$, $\mathrm{R}_{\mathrm{C}}=30 \mathrm{k} \Omega$. The $\mathrm{C}_{\mathrm{OVD} / \mathrm{S}}>\mathrm{C}_{\mathrm{ch}}$ due to a longer overlap length than the channel and the value of $\mathrm{g}_{\mathrm{m}}$ mentioned above is extracted from S21 directly, which is consistent with that of $\mathrm{g}_{\mathrm{m}}$ from a static I-V measurement $(\sim 16.4 \mu \mathrm{S})$.

The measurement results show a cutoff frequency of 3.11MHz while the proposed TFT and CMOS models predict cutoff frequencies of $3.14 \mathrm{MHz}$ and $2.72 \mathrm{MHz}$, respectively. This yields an error of $1 \%$ and $12.5 \%$, respectively.

Note that $\mathrm{H}_{21}$ can be calculated from all four s-parameters using the following relation,

$$
\mathbf{H}_{21}=-\frac{2 S_{21}}{\left(1-S_{11}\right)\left(1+S_{22}\right)+S_{12} S_{21}} .
$$

However, since the TFT is very resistive in s-parameter measurements, Eq.(8) can be approximated as,

$$
\left|\mathbf{H}_{21}\right| \approx\left|\frac{S_{21}}{s_{11}-1}\right| \text {. }
$$

Therefore, the frequency response of the TFT is dominated by the $S_{11}$ and $S_{21}$. The measurement and computational results 


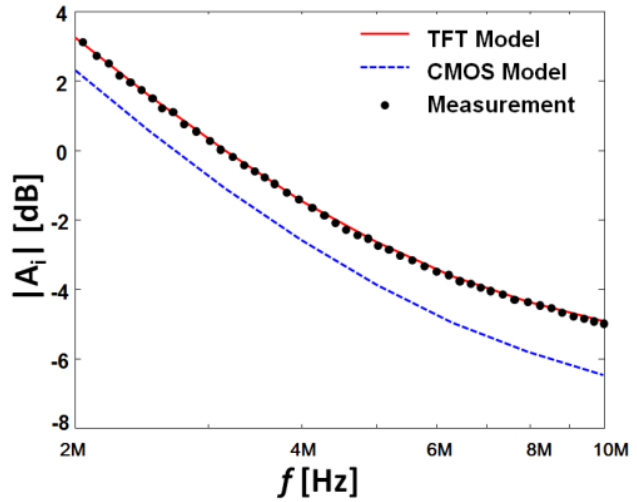

Fig. 3. Short-circuit current gain $\left(A_{i}\right)$ for the TFT with channel width $(W)=$ $100 \mu \mathrm{m}$ and channel length $(\mathrm{L})=10 \mu \mathrm{m}$, which is converted from the S-parameter measurement.
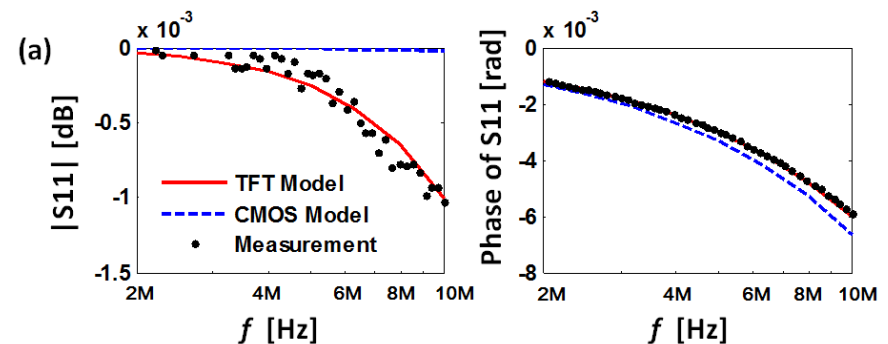

(b)
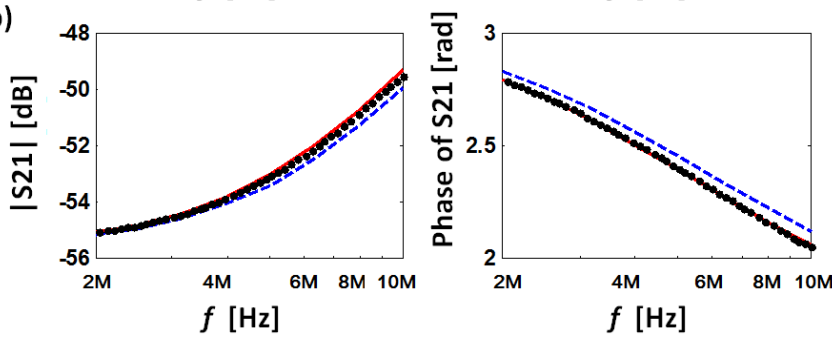

Fig. 4. (a) Amplitude and phase plots for $S_{11}$ and (b) those of $S_{21}$, respectively. Here, the measurements are compared with the computational results for the CMOS model and TFT model, respectively.

shown in Fig. 4 indicate that the major difference of the two models lies in $\mathrm{S}_{11}$, which can be explained by the different $\mathrm{C}_{\mathrm{ch}}$ connection at the gate-source port.

Another important factor for TFTs is the threshold voltage shift $\left(\Delta \mathrm{V}_{\mathrm{T}}\right)$. In order to examine its influence on the small signal behavior, a constant bias stress measurement is performed on a $\mathrm{W} / \mathrm{L}=50 \mu \mathrm{m} / 10 \mu \mathrm{m}$ TFT with $\mathrm{V}_{\mathrm{GS}}=8 \mathrm{~V}$ and $\mathrm{V}_{\mathrm{DS}}=15 \mathrm{~V}$ for total 10 hours while $\mathrm{f}_{\mathrm{T}}$ and $\mathrm{V}_{\mathrm{T}}$ are measured at every logarithmic time interval (the I-V sweep is done to extract the value of $\mathrm{V}_{\mathrm{T}}$, which briefly disrupts the constant bias). The measurement result is shown in Fig 5. The $\Delta \mathrm{V}_{\mathrm{T}}$ increases to $+0.2 \mathrm{~V}$ in total after the 10 hours bias stress and the respective $\Delta \mathrm{f}_{\mathrm{T}}$ drops to $0.08 \mathrm{MHz}$ almost linearly. This is due to the relatively small $\Delta \mathrm{V}_{\mathrm{T}}$ compared with $\mathrm{V}_{\mathrm{GS}}$ which leads to first order approximation of Eq. (7). From Eq. (3) \& (7) we have:

$$
\begin{aligned}
\Delta \boldsymbol{f}_{T} & \approx-\frac{r_{o i}\left(2 R_{C}+r_{o i}\right)\left(C_{o V}+C_{c h}\right)}{2 \pi\left(g_{m i 0} C_{o V} R_{C} r_{o i}+\left(2 R_{C}+r_{o i}\right)\left(C_{o V}+C_{c h}\right)\right)} K^{\prime} \Delta V_{T} \\
& =\beta \Delta V_{T}
\end{aligned}
$$

where $\beta$ is the constant found to be about $-3.2 \times 10^{6}[\mathrm{~Hz} / \mathrm{V}]$ for the examined TFTs. Eq.(10) allows to estimate the shift in unity gain frequency with threshold voltage shift. Here, the coefficient $\beta$ will be different for different material- and process-based TFTs.

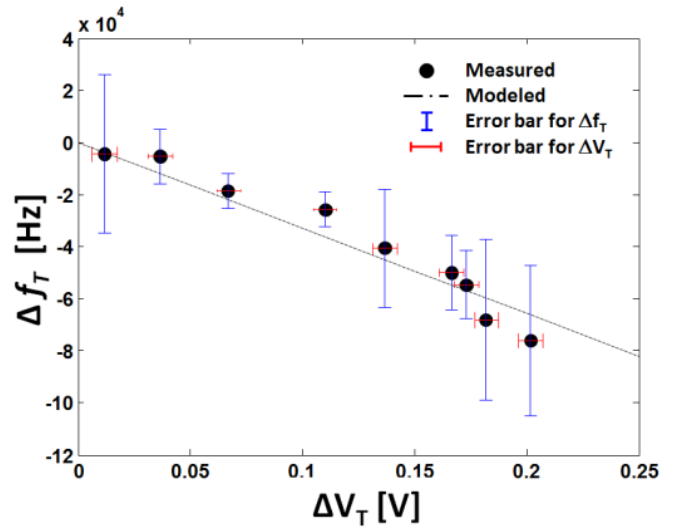

Fig. 5. Measured $\Delta \mathrm{f}_{\mathrm{T}}$ with respect to $\Delta \mathrm{V}_{\mathrm{T}}$ for the examined IGZO TFT.

Table I. Summary of parameters for the CMOS and TFT models

\begin{tabular}{|c|c|c|c|}
\hline Category & $\mathbf{R}_{\mathrm{C}}=\mathbf{0}$ & $\mathbf{C}_{\mathrm{ch}}<<\mathbf{C}_{\mathrm{ov}}$ & General case \\
\hline $\begin{array}{c}\text { CMOS and } \\
\text { TFT model } \\
\text { equivalence }\end{array}$ & Equivalent & Equivalent & Not equivalent \\
\hline $\begin{array}{c}\text { Parameter } \\
\text { relation }\end{array}$ & $g_{m}=g_{m i}=\frac{d I_{D S}}{d V_{\mathrm{GS}}}$ & $g_{m}=\frac{d I_{D S}}{d V_{\mathrm{GS}}}$ & $g_{m i}=\frac{g_{m} r_{o}}{r_{o}-g_{m} r_{o} R_{C}-2 R_{C}}$ \\
& $r_{o}=r_{o i}=\frac{d V_{D S}}{d I_{D S}}$ & $r_{o}=\frac{d V_{D S}}{d I_{D S}}$ & $r_{o i}=r_{o}-g_{m} r_{o} R_{C}-2 R_{C}$ \\
\hline $\begin{array}{c}\text { Cut-off } \\
\text { frequency }\end{array}$ & $f_{T}=\frac{g_{m}}{2 \pi C_{t o t}}$ & $f_{T}=\frac{g_{m}}{2 \pi\left(C_{t o t}-g_{m} R_{C} C_{c h}\right)}$ \\
\hline $\begin{array}{c}\mathrm{V}_{\mathrm{T}} \text { shift } \\
\text { terms }\end{array}$ & \multicolumn{2}{|c|}{$g_{m i}=g_{m i 0}-K^{\prime} \Delta V_{T}$} & $K^{\prime} \approx \frac{g_{m i 0}}{V_{G S}-V_{T 0}}$ \\
\hline
\end{tabular}

The summary of the model equivalence and parameter relations is given in Table I. Note that when CMOS model is used, the effect of $R_{C}$ is included in $g_{m}$ term. When the equivalent conditions are met, the TFT model yields to the CMOS model in all frequency range. The significance of the model difference is determined by value of $\mathrm{R}_{\mathrm{C}}$ and $\mathrm{C}_{\mathrm{ch}}$.

\section{CONCLUSION}

Small signal models are indispensable for the design of analog circuits, especially in the former for the correct phase margin and bandwidth of amplifiers and analog filters. This work reports on an accurate small signal model that takes into account the contact resistance, parasitic capacitance, and threshold voltage shift, while introducing internal transconductance $\left(\mathrm{g}_{\mathrm{mi}}\right)$ and output resistance $\left(\mathrm{r}_{\mathrm{oi}}\right)$. The proposed TFT model yields a $1 \%$ error in predicting the unity gain frequency, in contrast to $12.5 \%$ error using the CMOS model. It also provides a better fit to the measured s-parameters.

Theoretical analysis suggests that accuracy improvement stems from $\mathrm{C}_{\mathrm{ch}} \& \mathrm{R}_{\mathrm{C}}$ connection in the TFT model. It also suggests that the CMOS model can be inaccurate especially when the channel capacitance is more dominant and the contact resistance $\left(R_{C}\right)$ is bigger. The increase of $R_{C}$ due to down scaling of TFTs indicates that the proposed TFT model can be more beneficial to use in smaller devices. As $R_{C}$ and its separation of channel capacitance and overlap capacitance generally exist in many other types of device structures and material families, the model is potentially applicable to other TFTs fabricated on insulator substrate such as glass and plastic. Additional bulk parasitics should be considered when modelling TFT on a semiconductor substrate. 


\section{REFERENCES}

[1] D. Lovelace, J. Costa, and N. Camilleri, "Extracting small-signal model parameters of silicon MOSFETtransistors," 1994 IEEE MTT-S Int. Microw. Symp. Dig. (Cat. No.94CH3389-4), pp. 865-868, 1994.

[2] L. J. Giacoletto, "Diode and transistor equivalent circuits for transient operation," IEEE J. Solid-State Circuits, vol. 4, no. 2, pp. 80-83, Apr. 1969.

[3] P. Roblin, S. C. Kang, and W. R. Liou, "Improved Small-Signal Equivalent Circuit Model and Large-Signal State Equations for the MOSFET/MODFET Wave Equation," IEEE Trans. Electron Devices, vol. 38, no. 8, pp. 1706-1718, 1991.

[4] A. S. Sedra and K. C. Smith, Microelectronic circuits, 6th ed. New York Oxford University Press, 2011.

[5] B. Razavi, Design of Analog CMOS Integrated Circuits. McGraw-Hill, 2001

[6] P. R. Gray, P. Hurst, R. G. Meyer, and S. Lewis, Analysis and design of analog integrated circuits. Wiley, 2001.

[7] H. Marien, M. Steyaert, E. van Veenendaal, and P. Heremans, "Analog techniques for reliable organic circuit design on foil applied to an 18dB single-stage differential amplifier," Org. Electron., vol. 11, no. 8, pp. 1357-1362, Aug. 2010.

[8] S. Sambandan, "High-gain amplifiers with amorphous-silicon thin-film transistors," IEEE Electron Device Lett., vol. 29, pp. 882884, 2008.

[9] P. Bahubalindruni, V. G. Tavares, P. G. De Oliveira, P. Barquinha, R. Martins, and E. Fortunato, "High-gain amplifier with n-type transistors," 2013 IEEE Int. Conf. Electron Devices Solid-State Circuits, EDSSC 2013, vol. 2, no. 2, 2013.

[10] A. a. Fomani and A. Nathan, "Metastability mechanisms in thin film transistors quantitatively resolved using post-stress relaxation of threshold voltage," J. Appl. Phys., vol. 109, no. 8, p. 084521, 2011. P. Servati, D. Striakhilev, and A. Nathan, "Above-threshold parameter extraction and modeling for amorphous silicon thin-film transistors," IEEE Trans. Electron Devices, vol. 50, no. 11, pp. 2227 2235, Nov. 2003.

[12] M. J. Powell, "The physics of amorphous-silicon thin-film transistors," IEEE Trans. Electron Devices, vol. 36, no. 12, pp. $2753-$ 2763, 1989.

[13] P. V. Necliudov, M. S. Shur, D. J. Gundlach, and T. N. Jackson, "Modeling of organic thin film transistors of different designs," $J$. Appl. Phys., vol. 88, no. 11, p. 6594, 2000.

[14] H. Klauk, G. Schmid, W. Radlik, W. Weber, L. S. Zhou, C. D. Sheraw, J. A. Nichols, and T. N. Jackson, "Contact resistance in organic thin film transistors," Solid-State Electron., vol. 47, pp. $297-$ 301, 2003.

[15] P. V Necliudov, M. S. Shur, D. J. Gundlach, and T. N. Jackson, "Contact resistance extraction in pentacene thin film transistors," Solid. State. Electron., vol. 47, no. 2, pp. 259-262, Feb. 2003.

[16] S. J. Zilker, C. Detcheverry, E. Cantatore, and D. M. De Leeuw, "Bias stress in organic thin-film transistors and logic gates," Appl. Phys. Lett., vol. 79, no. 8, pp. 1124-1126, 2001.

[17] H.-W. Zan and S.-C. Kao, "The Effects of Drain-Bias on the Threshold Voltage Instability in Organic TFTs," IEEE Electron Device Lett., vol. 29, no. 2, pp. 155-157, Feb. 2008.

[18] S. Lee, A. Nathan, S. Jeon, and J. Robertson, "Oxygen Defect-Induced Metastability in Oxide Semiconductors Probed by Gate Pulse Spectroscopy," Sci. Rep., vol. 5, no. October, p. 14902, 2015.

[19] R. B. M. Cross and M. M. De Souza, "Investigating the stability of zinc oxide thin film transistors," Appl. Phys. Lett., vol. 89, no. 26, p. 263513, 2006.

[20] A. Suresh and J. F. Muth, "Bias stress stability of indium gallium zinc oxide channel based transparent thin film transistors," Appl. Phys. Lett., vol. 92, no. 3, p. 033502, 2008.

[21] J. E. Brittain, "Thevenin's theorem," IEEE Spectr., vol. 27, no. 3, p. 42, Mar. 1990

[22] D. H. Johnson, "Origins of the equivalent circuit concept: The current-source equivalent," Proceedings of the IEEE, vol. 91, no. 5. pp. 817-821, 2003.

[23] S. Lee, S. Jeon, R. Chaji, and A. Nathan, "Transparent semiconducting oxide technology for touch free interactive flexible displays," Proc. IEEE, vol. 103, no. 4, pp. 644-664, 2015.
[24] S. Lee and A. Nathan, "Conduction Threshold in Accumulation-Mode InGaZnO Thin Film Transistors," Sci. Rep., vol. 6, no. October 2015, p. 22567, 2016.

[25] S. Lee and A. Nathan, "Localized tail state distribution in amorphous oxide transistors deduced from low temperature measurements," Appl. Phys. Lett., vol. 101, 2012.

[26] S. Lee, A. Nathan, Y. Ye, Y. Guo, and J. Robertson, "Localized Tail States and Electron Mobility in Amorphous ZnON Thin Film Transistors," Sci. Rep., vol. 5, p. 13467, 2015. 